\title{
Contextual control of letter perception
}

\author{
ROHN J. PETERSEN and DAVID LaBERGE \\ University of Minnesota, Minneapolis, Minnesota 55455
}

\begin{abstract}
This study examined the hypothesis that the type of list context in which a given item is embedded may affect the way in which it is perceptually processed. In the first experiment, subjects matched four types of test items inserted in four types of lists. The types of test items and the types of lists in which they were embedded were two-letter words, two-letter spelling clusters, unrelated letter pairs, and pairs of novel letters. The first experiment indicated that only unrelated letter pairs were significantly affected by type of list in which they were inserted. The pattern of the effect was confirmed in the latency data of the second experiment and the interpretation was that only unrelated letter and related letter-group contexts were operating in the first two experiments. The third and fourth experiments were concerned with contrasting contexts at the feature and letter levels of processing. By using lists in which type of eatch trial either emphasized local features or global aspects of a pattern, the processing times of both familiar and unfamiliar letters were affected. The results of these experiments suggest that information processing models should incorporate structures which represent the way that context selectively facilitates processing of items at particular levels.
\end{abstract}

The purpose of this study was to investigate the way context interacts with specific stimulus inputs to influence the perception of short strings of letters. A string of letters forming a common word may be optionally perceived as one unit, as a series of syllable or spelling pattern units, or as a collection of letter units, according to the assumptions in many investigations. These investigations range from experiments dealing with the word advantage effect (e.g., Baron \& Thurston, 1973; Estes, 1975; Reicher, 1969; Wheeler, 1970) to theoretical descriptions of the reading process (e.g., Gibson \& Levin, 1975; LaBerge \& Samuels, 1974).

Recently this assumption has been set forth explicitly in terms of hierarchical models (Estes, 1975; LaBerge \& Samuels, 1974) in which the processing of a stimulus pattern involves feature analyzers, letter codes, spelling pattern codes, word codes, and word-group codes. These structures represent the memory of the subject's previous experience with the patterns. When a word is presented to the subject, it is assumed that outputs from lower levels, such as letter codes and spelling patterns, converge to activate a word code. It is the output from this word code that is sent to higher systems, which in turn operate upon it to elicit meaning and to produce an overt response. However, even though the presentation of a word may automatically activate a word code (LaBerge \& Samuels, 1974), it is conceivable that under some circumstances the subject may base his responses

This research was supported by United States Public Health Service Grants HD-06730 and MH-16270-04 and in part by the Center for Research in Human Learning through National Science Foundation Grant G13-35703X. The authors wish to thank Lee Brownston, Diana Randall, Ken Schultz, Dan Ansel, Dean Dreblow, and Mike Norden for their assistance in many phases of the study. We would also like to thank William Shaffer for his assistance with the data analysis. on outputs from codes at a lower level, for example, the component letters of the word.

One way to shift the level at which a string of letters is processed is illustrated in a word advantage type of study by Aderman and Smith (1971), in which subjects were led to expect to receive strings of unrelated letters in one condition and strings containing familiar spelling patterns in another condition. One group of subjects was given a list of 15 strings containing spelling patterns, followed by a trial in which the string contained unrelated letters. The other group was given a list of 15 strings of unrelated letters, followed by a string of spelling patterns. The results from the last two trials indicated that letters were detected more accurately in a spelling pattern string only when the preceding list contained spelling patterns. When the preceding list contained unrelated letter strings, no difference in accuracy was found. An additional finding was that the latency of letter recognition was the longest for the unrelated letter string tested at the end of the list of spelling patterns. The other three latencies were not significantly different from each other. Apparently, a given type of letter string was processed differently according to which kind of list preceded it. These findings could be interpreted as indicating that a given letter string was being perceived as a series of letters in one condition and as a group of spelling patterns in the other. Presumably, the subjects could take advantage of the information in the higher order units only when they were expecting these units.

The possibility that subjects may process the same pattern in different ways is an intriguing notion and warrants further experimental elaboration. In the first experiment to be reported here, the level of processing of strings of letters was manipulated by varying the predominant type of item in a list. The present procedure employed a simple matching task. The strings 
contained two letters and were of four types: words, spelling clusters, unrelated letters, and pairs of artificial letters.

\section{EXPERIMENT 1}

\section{Method}

Stimuli. The stimuli were lowercase letters and letter-like patterns grouped in pairs and arranged horizontally. A display always contained two pairs with five spaces between the pairs. When the pairs of patterns matched, the display was termed a target. When the pairs did not match, the display was termed a catch. The display pairs were selected from four lists: One list contained common two-letter words, and members of this list will be referred to as words; a second list was made up of consonant digraphs and blends that commonly occur at the beginning of English words, termed clusters. A third list was generated by recombining the individual letters used in the cluster list to form unfamiliar pairs of letters, termed letters. When these combinations were constructed, care was taken to assure than gross orthographic contours matched those of the cluster list (e.g., ascender and descender combinations were matched). The fourth list contained pairs of artificial letters that were termed features.

In order to probe the effects of various list contexts on target items, two test sets of four items were selected from each of the four types of lists. The items selected from the cluster list were combinations of the consonants used in the word test sets. The two letter test sets were formed by recombining the individual letters of each of the cluster test sets. The orthographic contours of each letter set matched those of a corresponding cluster set. In the feature test sets, the items were constructed to match

\begin{tabular}{|c|c|c|c|}
\hline Words & Clusters & Letters & Features \\
\hline & \multicolumn{3}{|c|}{ SETI } \\
\hline it & th & ht & ลว \\
\hline by & fl & $f b$ & $7 p$ \\
\hline he & sw & rw & rلs \\
\hline or & br & Is & 12 \\
\hline \multicolumn{4}{|c|}{ SET 2} \\
\hline if & wh & hs & $\lambda_{2}$ \\
\hline we & $\mathrm{fr}$ & wl & آل \\
\hline 10 & st & $\mathrm{rb}$ & $r p$ \\
\hline us & bl & tf & 77 \\
\hline \multicolumn{4}{|c|}{ FILLER ITEMS } \\
\hline as & ch & rc & $r 7$ \\
\hline ho & $\mathrm{dr}$ & hd & yd \\
\hline in & $\mathrm{gl}$ & hg & ते \\
\hline me & ph & $1 p$ & $1 \mathrm{p}$ \\
\hline on & tw & $r i$ & rt \\
\hline an & $\operatorname{tr}$ & Ih & ה \\
\hline do & sk & ts & $\ngtr_{2}$ \\
\hline so & $\mathrm{cl}$ & kc & +7 \\
\hline $\mathrm{ma}$ & $g r$ & wg & 25 \\
\hline ha & sh & rs & +2 \\
\hline
\end{tabular}

Figure 1. Stimuli used in Experiments 1, 2, and 3. those in the letter and cluster test sets in terms of gross orthographic contour. Finally, a set of 10 filler items was selected from each list. The test items and filler items are shown in Figure 1. Catch displays were constructed by selecting two different items from the same list and presenting them as nonmatching pairs. Catch displays were balanced with respect to number of letters in common and the position of the common letter. Half of the catch trials had the same contour for both pairs; half had different contours.

Apparatus. The displays were presented on a $38-\mathrm{cm}$ diagonal television screen from a Tektronix 4501 scan converter. A Data General 1220 computer controlled the presentation of stimuli and recorded the subjects' responses. Each letter was conscribed within a 7 by 15 dot matrix that measured $11 \times 23 \mathrm{~mm}$ on the screen. The pairs of letters were separated by $7.8 \mathrm{~cm}$, corresponding to 5 spaces on the screen. The display subtended an angle of $2.97 \mathrm{deg}$.

Two subjects were tested concurrently in separate dimly-lit rooms. The television screen was mounted at eye level, $125 \mathrm{~cm}$ from the edge of the table where the subject was seated. The response button was $25 \mathrm{~mm}$ in diam and required a $2-\mathrm{mm}$ downward displacement to close a microswitch. The button was mounted on an inclined plane and positioned on the table in front of the subject.

Procedure. A trial began with the presentation of a small circle centered in the upper part of the screen for $750 \mathrm{msec}$. The small circle served as a warning signal. After a $750-\mathrm{msec}$ blank period, the display appeared for $1,000 \mathrm{msec}$. The time between a display and the next warning signal was $800 \mathrm{msec}$. The subject was instructed to respond when the pairs of items in the display matched, but to withhold his response when they did not match. Responses were recorded by the computer up to $1,500 \mathrm{msec}$ after the onset of the display. Target exposures were terminated by a response; catch displays always remained exposed for 1,000 msec. When the subject responded to a catch display, a red light located $40 \mathrm{~mm}$ above the center of the screen flashed for $450 \mathrm{msec}$.

Subjects. Forty experimentally naive undergraduates at the University of Minnesota served as subjects. They were given two class points for their participation.

Design. There were four conditions in the experiment. Each condition corresponded to one of the four types of lists shown in Figure 1. There were 10 subjects in each of the four conditions. Each subject was given only one type of list condition. Each condition contained three blocks of trials; the first block was a practice block of 30 trials, and the other two were test blocks of 90 trials each. Items from the filler set were used for the practice block, and each display was repeated so that there were 20 target displays and 10 catch displays. The first test block was constructed by combining a filler set with test sets from each of the four types of lists. For a given condition the set of 10 filler items was augmented by adding four items from the other test set in that condition. For the second test block these additional four items were used as the test items and the four test items in the first block were added to the filler items. The remaining test items were chosen from the other three conditions, and a different set of these test items was used in each test block. The order of presentation of the two sets of test items was balanced across subjects. The test items and the four additional filler items appeared only once in a block.

A test block of 90 items contained 76 target displays and 14 catch displays. Each of the 10 filler items from a list in Figure 1 was used four times, and each of the additional fillers from a test set was used once, for a total of 44 target trials. Catch displays were chosen in a similar manner so that 20 came from the filler list and 2 from the test set. The test trials contained four target and two catch displays from each list, for a total of 16 target trials and 8 catch trials. Thus, a test block consisted of 24 test items and 66 filler items. Since six of the test items were from the same type of list as the filler items, there was a total of 


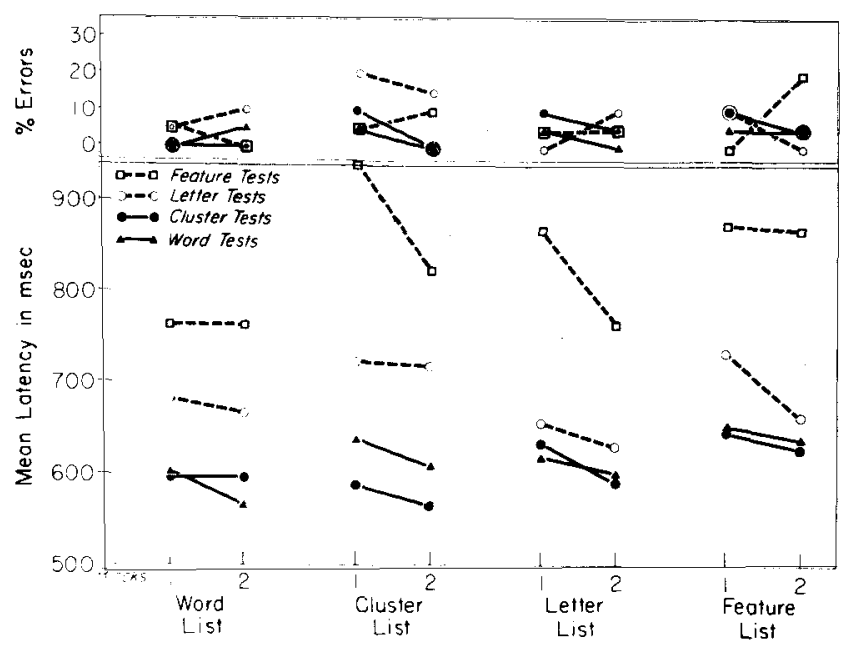

Figure 2. Mean latency and percent errors of matching responses from Experiment 1 for four types of test items as a function of type of item which predominated in a list. Patterns were presented simultaneously.

72 displays in a block of 90 trials which were of the same type. Therefore, there was an $80 \%$ chance of receiving an item of one particular type, which served as the means of generating a context within a block.

All trials were presented in a random order, with the following restrictions for the two test blocks: The first six trials in a block were filler items; four of these were target displays, and two were catch displays. Test trials were separated by at least two filler trials.

\section{Results}

The mean latencies and percent errors of responses to matching pairs of items are shown in Figure 2. Latencies for the four types of test displays are plotted as a function of the two test blocks for each of the four list conditions. Only significant and marginally significant Fs are reported. An analysis of variance of the latency measures revealed a significant main effect for (1) type of test item $[\mathrm{F}(3,108)=151.36, \mathrm{p}<.01, \mathrm{MSe}=5,959.35]$ and (2) replication of test blocks $[F(1,36)=9.46$, $\mathrm{p}<.01, \mathrm{MSe}=3,941.89]$. The only significant interaction involved Type of Test Item by List Type, $[F(9.108)=3.34, p<.01, \mathrm{MSe}=5,959.35]$. The details of this relatively global interaction were probed with selected Scheffé post hoc comparisons of test item latencies within the four types of lists.

Within the word list, there was no significant difference between cluster and word items. Letter items were significantly different from cluster and word items $[\mathrm{F}(3,108)=5.43, \mathrm{p}<.01, \mathrm{MSe}=5,959.35]$, and feature items were significantly different from letter, cluster, and word items $[\mathrm{F}(3,108)=17.63, \mathrm{p}<.01$, MSe $=$ 5.959.35].

Within the cluster list, no significant difference was found between word and cluster items. Letter items were significantly greater than cluster and word items
$[F(3,108)=11.43, p<.01, \mathrm{MSe}=5,959.35]$. Feature items were significantly different from letter, cluster, and word items $[\mathrm{F}(3,108)=53.66, \mathrm{p}<.01, \mathrm{MSe}=$ $5,959.35]$.

Within the letter list, no significant difference was found between word and cluster items, nor were letter items significantly different from cluster and word items. However, feature items were significantly greater than letter, cluster, and word items $[\mathrm{F}(3,108)=31.95$, $\mathrm{p}<.01, \mathrm{MSe}=5,959.35]$.

Within the feature list, no significant difference was found between word and cluster items, and letter items were not significantly different from cluster and word items. Feature items were significantly greater than letter, cluster, and word items $[F(3,108)=36.65$, $\mathrm{p}<.01, \mathrm{MSe}=5,959.35]$.

An analysis of variance of error data showed no significant main effects or interactions.

\section{DISCUSSION}

The results of this experiment revealed that the rates of processing word and cluster items were not differentiated by manipulating list context. Furthermore, the rate of processing feature items relative to word, cluster, and letter items' latencies did not change as a function of list context. List context appeared to influence only the rate of processing letter items relative to word and cluster items. When the context list comprised word or cluster items, letter items were processed substantially slower than either words or clusters. When the context list comprised letter or feature items, letter items were processed at a rate similar to that of word and cluster items.

\section{EXPERIMENT 2}

In order to establish more firmly that list context affects processing of letter pairs, the design was simplified to include only two types of lists and two types of test items.

\section{Method}

Stimuli. The display items were selected from the cluster and letter lists shown in Figure 1, and presented in the same positions on the television screen.

Apparatus and procedure. These were similar to Experiment 1 , except that the durations of trial events were reduced slightly to increase the speed of data gathering.

The warning signal was exposed for $500 \mathrm{msec}$, followed by a blank for $500 \mathrm{msec}$. The display was exposed for $1,000 \mathrm{msec}$ and the blank intertrial interval was $500 \mathrm{msec}$. Responses were accepted by the computer up to $1,250 \mathrm{msec}$ after the onset of the display.

Subjects. Twenty undergraduate students volunteered and were given two class points for their services.

Design. Each of the two list conditions, clusters and letters, had 10 subjects. Except for a change in the number of test trials, the content of the trial blocks was approximately the same 


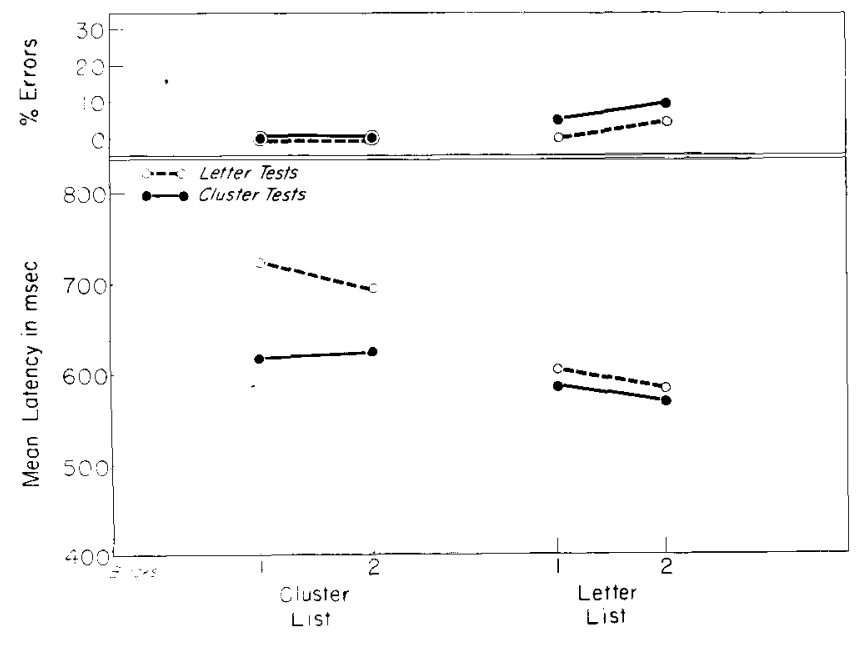

Figure 3. Mean latency and percent errors of matching responses from Experiment 2 for cluster and letter test items as a function of the type of context list.

as in Experiment 1. There were three blocks: one practice and two test blocks. The practice block contained 48 trials. The block was constructed of 32 filler target displays and 16 filler eatch displays. Eight of the 10 filler items of a list in Figure 1 were used three times, and two were used four times. The 16 filler catch items were constructed in a similar manner. Test blocks contained 60 trials, and were constructed by merely adding four test items from the cluster lists and four test items from the letter list plus two catch items constructed from each list. Displays were randomized within a block subject to the same restrictions as in Experiment 1.

\section{Results}

Shown in Figure 3 are the mean latencies and percent errors of responses to matching pairs of items. An analysis of variance showed significant main effects for type of test item $[F(1,18)=11.99, \mathrm{p}<.01, \mathrm{MSe}=$ $4,647.99]$ and list type $[\mathrm{F}(1,18)=9.25, \mathrm{p}<.01$, MSe $=12,479.21]$. The only significant interaction was Type of Test Item by List Type $[\mathrm{F}(1,18)=5.36, \mathrm{p}<.05$, MSe $=4,647.99$ ].

The interaction was probed by Scheffé post hoc comparisons of test items in each list. The latency to letter items changed significantly as a function of list type $[F(1,18)=27.61, p<.01, \mathrm{MSe}=4,647.99]$. No difference was found for cluster items in the two lists. Inspection of Figure 3 reveals the same pattern of results found in Figure 2 for the letter and cluster items in letter and cluster lists. An analysis of variance of the error data indicated a significant main effect only for list type $[\mathrm{F}(1,18)=6.00, \mathrm{p}<.05, \mathrm{MSe}=3.33]$, but the size of the effect was small.

\section{DISCUSSION}

The pattern of results replicated the finding in Experiment 1 with respect to the effect of letter and cluster items in letter and cluster lists. The elevation of lette1 items in cluster lists is a result similar to that found by Aderman and Smith (1971), who measured latency of letter identification in similar kinds of lists in the Reicher-Wheeler forced-choice test.

The interpretation favored here of the effect of list context upon matching letter bigrams is that a change in type of list induces a change in the level of processing of the bigrams. More precisely, list changes are assumed to affect the level of codes from which readouts are sent to the matching operations stage. This allows for the possibility that more than one level of perceptual codes may be activated by a stimulus display. The critical issue for the present set of experiments is concerned with which level of codes is used to perform a matching operation and subsequently produce an overt response.

It seems clear that matching operations can be made not only on the basis of visual information, but also on phonological or on semantic information as well. Posner and Mitchell (1967) induced subjects to match letters either on the basis of physical (visual) properties or naming (phonological) properties or categorical (semantic) properties by use of appropriate catch trials. For the present experiments, we should examine the possibility that matching letter bigrams may take place not only on the outputs of visual codes, but also on the outputs of phonological codes, and perhaps even of semantic codes.

We are inclined to rule out the possibility of semantic matches in Experiment 2 because the stimulus items have neither meaning nor any apparent basis for categorization other than familiarity when considered as clusters. In Experiment 1, however, some of the lists and test bigrams were words, so that a meaningful vs. nonmeaningful categorization was possible as a basis for matching. If matching were carried out in this way, then the results in the word list of Figure 2 should show a difference between word tests and cluster tests, and no difference between feature, letter, and cluster tests. However, since this was not the obtained pattern of findings, we conclude that semantic processing was not the basis of matching even in the word list of Experiment 1 .

We are, therefore, apparently left with the consideration of the phonological and visual modes or, possibly, some combination or mixture of the two. By assuming the conceptual framework described by LaBerge and Samuels (1974), we infer that phonological processing is contingent upon processing the item at a visual level. Let us assume that only visual letter codes are read out into the phonological system. Then the pattern of results shown in Figure 3 could have resulted from phonological matches in the following way. In the letter list condition, the subject matches the names of the letters. Thus, the cluster and letter test items do not differ in mean latencies. In the cluster list condition, the visual letter outputs are assumed to be unitized at 
the phonological level into a single-syllable sound. This occurs automatically for the familiar clusters but not for the unfamiliar letter test items. Letter test items require longer time to match either because attention is needed to unitize them into a syllable or because attention takes time to switch to the letter name level and matching is carried out at the component level.

The second way that phonological matching may be related to the pattern of results assumes that visual cluster codes are always read out into the phonological systems (cf. LaBerge \& Samuels, 1974). Here they activate syllable-like units. In the cluster list condition, familiar phonological units will be processed automatically, but the unfamiliar units will take additional time for unitization, or for attention to switch to the letter sound or letter name level. In the letter list condition, the subject always decomposes the syllable into component letter names or letter sounds and matches these. Thus, familiar clusters and unfamiliar letter test items would be treated alike and yield equivalent mean latencies.

Therefore, regardless of which of the two ways that phonological units are assumed to be activated from the visual system, the list context conditions selectively affect the level at which phonological matching takes place.

The interpretation of matching visual codes is relatively straightforward. According to the LaBergeSamuels (1974) hierarchical model, the familiar pattern ch activates the unitary code ch at the cluster level and also the component letter codes $c$ and $h$ at the letter level. In the letter list condition, the match is always made in terms of letter codes, and therefore both letter and cluster test items are processed in the same way and with equivalent latencies. In the cluster list condition, the familiar cluster test items are unitized automatically, but the unfamiliar letter test items require additional processing time, either to allow attention to unitize them prior to matching or to allow time for attention to switch to the letter codes for matching. We have already considered the combination of visual and phonological letter names.

Thus, both visual and phonological matching interpretations of the present experiments are possible under assumptions of the LaBerge-Samuels (1974) theory of information processing. What is new is the theoretical requirement that some mechanism determine for a given stimulus display what system, and at what level within that system the codes will be read out for an operation such as matching. For example, when a familiar bigram such as ch is visually presented, many codes are assumed to be activated automatically, namely, the visual letter codes and their associated name codes and the visual cluster code and its associated syllable code. How does the subject determine which of these four activated codes is sent on for further processing? For a discussion of possible mechanisms which could be added to a hier- archical model to provide this capability, the reader is referred to Estes (1975), LaBerge (1976, 1977), and LaBerge, Petersen, and Norden (1976).

However, before proceeding to the next experiments, it may be helpful to attempt to determine whether matching operations in the first two experiments were carried out on visual or on phonological code outputs. Our reasoning for this test hinges on the assumption that phonological matching in the cluster list condition should be faster for familiar syllables than for unfamiliar syllables. An independent indicator of this difference would be a direct test of pronounceability of the several test items. On the basis of the results shown in the cluster and word lists of Figure 2, we should expect that word and cluster test items should be pronounced with about the same latency but that letter test items should be pronounced with a substantially longer latency than either word or cluster test items.

We asked a group of 10 subjects to pronounce aloud a series of bigrams that were presented on a TV screen. The subject's task in this experiment was to pronounce the bigram as a unit and to respond by pressing a button as soon as he had completed pronouncing the item. The experimenter remained in the room to insure that the subject did indeed pronounce the item before the response was made. The stimuli were the test items from the word, cluster, and letter lists of Experiment 1, randomly arranged within a block of 48 trials. The filler items from these lists were presented in a warm-up block. The results of this experiment indicated that the basic assumption of the pronounceability hypothesis is correct: There was a stratification of latencies. The mean latency for the word items was $752 \mathrm{msec}$, for the cluster items, $802 \mathrm{msec}$, and for the letter items, $858 \mathrm{msec}$ $[\mathrm{F}(2,18)=10.14, \mathrm{p}<.01, \mathrm{MSe}=2,750.53]$.

Notice that the word and cluster test items showed about the same magnitude of difference as that of the cluster and letter test items in the pronunciation tests. If matching is based on pronounceability, then the results of Experiment 1 shown in Figure 2 should indicate faster matching latencies for word test items than for cluster test items in the word and cluster lists. However, this prediction is not supported by the data, since matching latencies of cluster and word test items were not significantly different under any of the conditions. These considerations cast doubt on the hypothesis that matching of these items was based on outputs from phonological codes. It seems reasonable, then, to infer that, in Experiment 1, the cluster condition induced the subjects to expect to match items on the basis of visual code outputs. How, then, do we interpret the finding that the differences in pronunciation latencies between the letter test items and the cluster test items were closely related to the difference between matching latencies between these same items shown in the cluster conditions of Figures 2 and 3? This correspondence between pronounceability and matching could be 
explained on the basis of visual code outputs alone by considering that both the pronununciation task and the matching task require that the visual cluster unit be processed first. If letter items require more time to unitize visually than the more familiar cluster items, then this difference would be revealed both when a matching task was performed on these outputs and when a pronunciation task was performed from the phonological units associated with these visual codes.

Taken together, then, the data from the pronunciations and matching tests seem to support the hypothesis that matching was performed on visual codes, not on phonological codes. The times required to activate visual word and familiar cluster units apparently are approximately the same, but the time to activate unfamiliar letter-pair units (by attentional unitization) is substantially longer. In fact, sometimes it may be so long as to require switching attention to a lower level where matching is carried out on letter components. It takes a longer time to pronounce clusters than words either because the association links from the visual codes to the phonological codes differ in strength and/or because the phonological codes differ in familiarity. The relatively long latencies of pronouncing unfamiliar letter pairs may be due not only to weak associative links from the corresponding visual codes, but also to the fact that the unfamiliar visual codes apparently require longer processing time.

Incidentally, when subjects were asked to describe what they thought they were doing in the matching task, they often mentioned that they were aware of the letter and cluster sounds. According to the LaBerge-Samuels (1974) theory, it would be expected that the association of letter names and cluster sounds would be activated automatically when the visual patterns are presented. Although the theory is not explicit with respect to the automaticity of matching operations, it seerns reasonable to expect that matching could take place simultaneously for both visual and phonological units. The critical question for these experiments is which matching operation first produces the buttonpress. The pronunciation and matching experiments presented above seem to indicate that the responses recorded were driven by outputs from visual matches. For other evidence that matching of graphemic stimuli can be carried out on a visual basis, the reader is referred to Baron and Thurston (1973) and Pollatsek, Well, and Schindler (1975).

It may seem somewhat surprising that well-learned patterns such as clusters were not processed significantly faster in cluster lists than in letter lists. Even if clusters are perceived as units in the cluster lists but as separate letters in the letter list, the difference between the times to make one match and two matches may be relatively small. Perhaps contrasting the latencies to patterns of three or four letters would provide a stronger test of this effect.

Considering Figure 2, it is clear by the consistently longer latencies for feature tests in all lists that these patterns take longer to process, but list context apparently did not have a clear differential effect. One might ask whether these longer latencies reflect a more complicated arrangement of features in the artificial letter patterns than in the familiar letter patterns. A study by LaBerge (1973) addressed the same question with respect to the artificial letters used to study perceptual learning. It was shown that the latency difference between the artificial letters and the real letters was zero when items were displayed successively for matching, but was about $48 \mathrm{msec}$ when the items were presented simultaneously. This difference gradually decreased across days of practice until it disappeared. It was therefore concluded that the unfamiliarity of a pattern affected its latency under simultaneous matching conditions but not under successive conditions. Thus, while the simultaneous matching procedure was adopted as a measure of familiarity, it contained a complexity component as well. The successive matching procedure, however, was assumed to be a measure of pattern complexity which was relatively free of familiarity factors. The latter measure was applied in the next experiment to determine whether the longer latencies for feature items in Figure 2 were due to greater pattern complexity.

\section{EXPERIMENT 3}

\section{Method}

Stimuli, apparatus, and design. These were exactly the same as in Experiment 1.

Procedure. Each trial began with the presentation of a pair of letters centered in the upper part of the screen. The subject was instructed to observe this stimulus but not to respond to it. After a short blank interval, an item appeared in the lower part of the screen. The subject was instructed to respond if this item matched the one he had just observed in the upper part of the screen. The two items chosen for successive presentation were the same items presented simultaneously in a display of Experiment 1 . The durations of events within a trial were the same as in Experiment 1.

\section{Results}

The mean latencies and percent errors of responses to successively presented pairs of letters are shown in Figure 4.

An analysis of variance of the latency data yielded significant main effects for (1) type of test item $[F(3,108)=13.39, \mathrm{p}<.01$, MSe $=2,198.60]$ and (2) replications of the test blocks $[\mathrm{F}(1,36)=9.24$, $\mathrm{p}<.01, \mathrm{MSe}=4,866.22]$. A significant interaction was found for Type of Test Item by List Type $[F(9,108)=$ $2.32, \mathrm{p}<.01, \mathrm{MSe}=2,198.60]$. As in Experiment 1, Scheffé post hoc comparisons were used to probe this interaction. Within-list comparisons yielded only one significant effect, namely, that feature test items had significantly longer latencies than word, cluster, and 


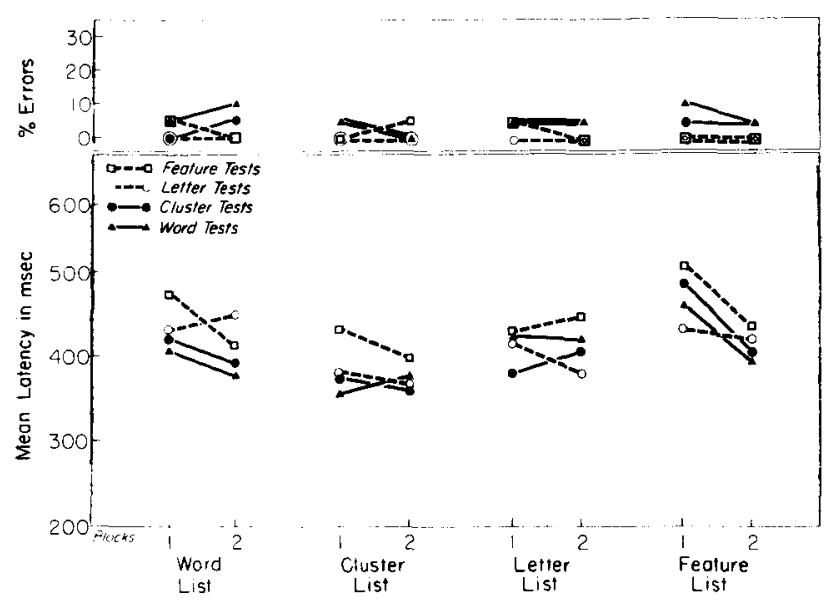

Figure 4. Mean latencies and percent errors of matching responses from Experiment 3 for four types of test items as a function of type of item which predominated in a list. Patterns were presented successively.

letter test items only in the cluster list $[\mathrm{F}(3,108)=4.86$, $\mathrm{p}<.01, \mathrm{MSe}=2,198.60]$. An analysis of variance of the error data revealed no significant differences.

\section{DISCUSSION}

The interaction of Type of Test Items by List Type appears to be due to the difference found for items in the cluster list, where feature items were slower than letter, cluster, and word items. The occurrence of this difference only in the cluster context is difficult to account for. If we accept these results as reflecting the relative complexity of items used in Experiment 1, then feature items may be considered more complex, but word, cluster, and letter items were not differentiated according to complexity. Although the feature items were $46 \mathrm{msec}$ slower than the other items in the cluster list, this difference is not large enough to allow us to assert that the substantially longer latencies to feature items in Experiment 1 were due entirely to the compleity of the patterns. Rather, it would seem that their longer latencies were due to unfamiliarity (LaBerge, 1973).

With regard to the effects of the feature list shown in Figure 2, one might ask whether the test patterns were being processed feature-by-feature or by some other, perhaps more global, procedure. While the evidence presented here is not conclusive concerning this question, it does seem unlikely that the feature list induced the subjects to process the familiar test patterns, namely, words and clusters, in a way which added substantially more time to their processing rates in other list contexts. If subjects were indeed attending to features in the feature list, they managed to shift to higher unit codes quite rapidly when a word or cluster item appeared. The simultaneous matching task used in Experiment 1 may well have provided an opportunity for this kind of switching of levels. Consider that when a pair of words is presented, the first item of the display is scanned at both the feature and word levels. As the scan moves to the second item, the subject may have time to switch his attention to the word level for purposes of matching the first item with the second item as a whole. In this way, the processing at the feature level which the subject may have initiated may have been obscured. This may also have been the case for processing clusters or words in the letter list. Given a word, subjects may indeed have started processing the first item of the display preparing to match letters, but shifted to the word level during the shift of scan to the second item of the display and matched words instead.

This line of reasoning is based on the assumption that a familiar pattern is processed automatically at the unit level as well as at subordinate component levels (LaBerge \& Samuels, 1974). All levels may then be read out into the matching operation simultaneously, and the first one to yield a match evokes the response. However, a particular level may be biased to read out more quickly when there are contextual factors such as the type of items the subject has been processing prior to the test item.

In view of these considerations, the failure to find a context effect in the feature list of Figure 2 may be ascribed to a failure to induce matching operations at the feature level in the feature list. The fourth experiment was directed toward establishing a mode of processing which was intended to induce the subject to compare features when he matched novel letters and when he matched familiar letters.

\section{EXPERIMENT 4}

The paradigm of Experiment 2 was employed, but the displays consisted of single letters instead of pairs of letters. The feature context was established by using catch displays in which the letters varied only slightly, as would typically occur in different typewriter fonts or handwriting. The letter context was established by using catch displays that consisted of two relatively unconfusable patterns.

\section{Method}

Stimuli. The items of a display were uppercase letters and letter-like patterns shown in Figure 5 . Each pattern was conscribed in an 11 by 11 dot matrix that measured $17 \times 17 \mathrm{~mm}$. The two letters of each display were separated by seven spaces on the television screen. The display subtended a visual angle of $5.23 \mathrm{deg}$. The display was generated by a computer through the scan converter, as described in Experiment 1.

Ten familiar letters were selected from the alphabet. Four of those letters were designated as test items and six as fillers. Ten artificial letters were constructed with some global similarity to the 10 letters of the alphabet. For each familiar and unfamiliar filler letter, four variations were constructed which differed in "script" style from the main letter items (cf. Figure 5).

Procedure. The procedure of this experiment was similar in all respects to that of Experiment 2. 


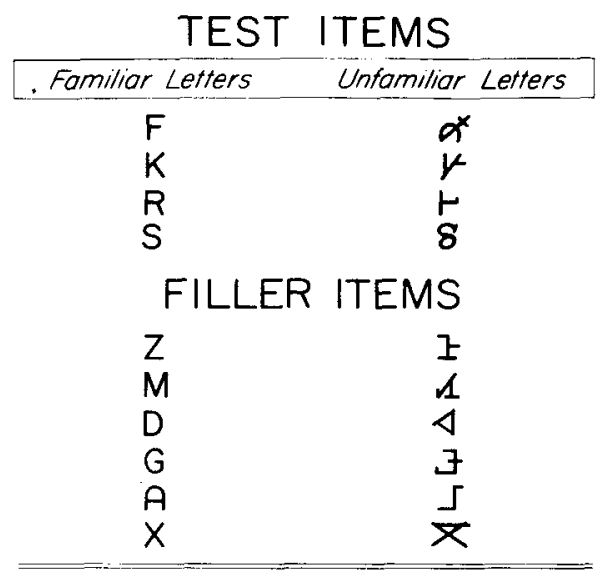

Examples of script difference catch displays

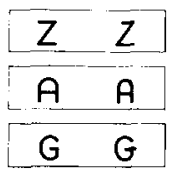

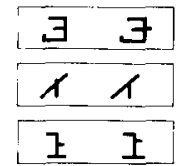

Examples of letter difference catch displays:

\begin{tabular}{|c|c|}
\hline$z$ & $x$ \\
\hline A & $D$ \\
\hline & $M$ \\
\hline
\end{tabular}

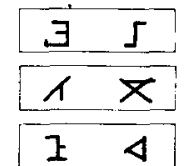

Figure 5. Stimuli used in Experiment 4 and examples of catch displays used to determine the context of a list.

Subjects. Twenty undergraduate volunteers from the intro. ductory psychology class were given $\$ 2$ or two class points for their services.

Design. There were 10 subjects in each of two list conditions. The lists differed with respect to the type of catch trial employed (LaBerge, 1971). For the feature list, the catch trial was always a change in "script" (cf. Figure 5); for the letters list, the catch trial was a different pattern within the main set of six familiar filler letters or six unfamiliar filler letters. Half of the filler items for each list were familiar letters, half unfamiliar letters. An equal number of familiar and unfamiliar letters was used in each list to give subjects enough exposure to unfamiliar letters so that they would not produce long latencies on test trials because of their novel category.

Each subject was given three blocks of trials, one practice block of 54 trials and two test blocks containing 66 trials each. The practice block contained 36 filler targets and 18 filler catches. Half of the trials were familiar letters and half were unfamiliar letters. A test block was constructed by adding 12 test trials to the filler trials of a practice block. Half the test trials were familiar letters, half unfamiliar letters, and of the six test trials of one type, four were targets and two were catch displays. The catch trials for test items were letter differences in both conditions (cf. Figure 5).

\section{Results}

Mean latency and percent errors of responses to the test displays are shown in Figure 6. An analysis of variance of the latency data yielded significant main effects for (1) type of catch trial $[F(1,18)=27.39$,

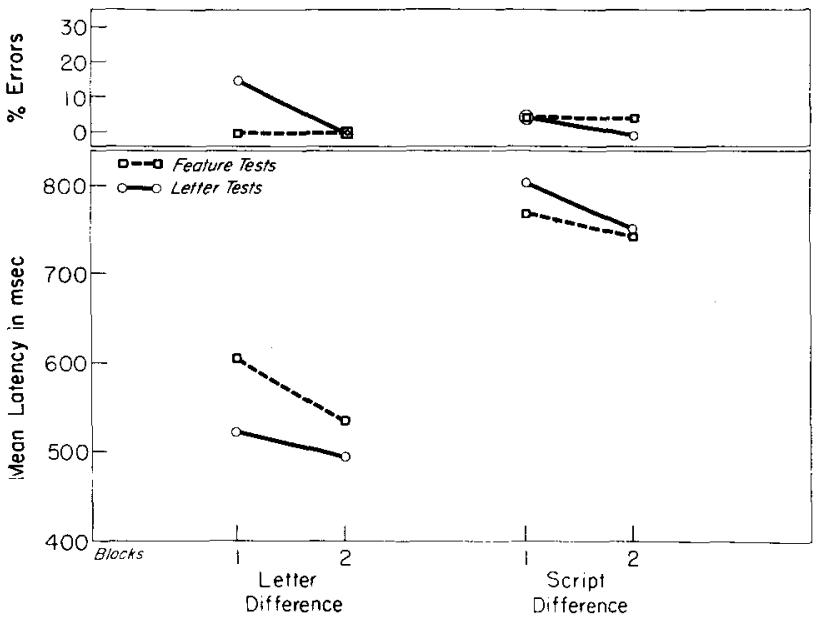

Figure 6. Mean latency and percent errors of matching responses from Experiment 4 for letter and feature test patterns as a function of the type of catch display used in a list. Patterns were presented simultaneously.

$\mathrm{p}<.01, \mathrm{MSe}=38,775.72]$ and $(2)$ replication of test blocks $[\mathrm{F}(1,18)=6.61, \mathrm{p}<.01, \mathrm{MSe}=5,433.54]$. The only significant interaction was Type of Catch Display (letter or script difference) by Type of Test Item $[F(1,18)=5.27, p<.05, \mathrm{MSe}=6,133.51]$. None of the error comparisons was significant.

The interaction of Type of Catch Display by Test Item was probed by Scheffé post hoc comparisons. For the script catch display condition, the familiar test item latencies were $20 \mathrm{msec}$ slower than the unfamiliar test item latencies, but this difference was not significant.

However, for the letter catch display condition, the familiar test item latencies were $59 \mathrm{msec}$ faster than the unfamiliar test item latencies, and this difference was significant $[F(1,18)=5.80, p<.05, \mathrm{MSe}=6,133.51]$.

\section{DISCUSSION}

The results of Experiment 4 clearly indicate that two letters, whether familiar or unfamiliar, are matched in a manner depending upon the kind of catch display used for the items in the list. Both familiar letter and unfamiliar feature displays were processed slower in the script condition than in the letter condition, suggesting that matching at the feature level takes longer than matching at the global letter level. The fact that there was no difference in processing rates for the two types of test items in the script condition supports the interpretation that both familiar and unfamiliar letters were processed at the same level, probably the feature level. Furthermore, under the letter condition, the familiar letters were processed faster than the unfamiliar letters, because the familiar letters presumably already had the benefit of a great many exposures in which perceptual learning had achieved unitary processing (LaBerge, 1973). The 
advantage possessed by familiar letters over unfamiliar letters, however, was not revealed under conditions in which the subject was induced to process a match at the feature level, such as in the feature condition.

The conclusions we have drawn from the latency data within a catch display condition are based on the assumption that any obtained differences are due to differences in level of processing and familiarity, and not to differences in the absolute degree of complexity of the patterns. In order to obtain an independent comparison of the complexity of the patterns used in Experiment 4 , we presented these patterns one at a time to a new group of 20 subjects, using the successive procedure described in Experiment 3. The mean latencies obtained over the two test blocks of the letter condition were $366 \mathrm{msec}$ for the letter tests and $348 \mathrm{msec}$ for the feature tests. For the script condition the comparable mean latencies were $518 \mathrm{msec}$ for the letter tests and $510 \mathrm{msec}$ for the feature tests. An analysis of variance of the latency data indicated no significant differences between letter and feature test items in either condition. As expected, the difference in latency across catch display conditions was highly significant. Since the familiar and unfamiliar patterns used in Experiment 4 were not shown to be different in complexity, the obtained difference between feature and letter tests under the letter condition in Figure 6 is assumed to be due to differences in familiarity of these patterns.

When the results in Figure 6 are compared with those in Figure 3, the interactive effect of test items with conditions is quite similar. The similarity of the interaction would support the notion that similar kinds of events are occurring in both experiments. The context of the task, whether established by type of filler list or type of catch display, apparently affects the way the items are processed. A perceptual hierarchy may be reflected by the latency differences between items that are familiar and unfamiliar at a given level of the hierarchy. Familiar and unfamiliar items are not differentiated when the context establishes processing of both items at lower levels, where component codes are represented. When context establishes processing at a higher level, where wholistic codes are represented, the items are differentiated with respect to familiarity, with the familiar items being processed faster than the unfamiliar items.

By inspection of Figures 6 and 3, we can follow the development of a perceptual hierarchy. The lowest level contains feature analyzers and is reflected in the results of the script condition in Figure 6, where familiar letter patterns and unfamiliar letter patterns are processed at the same rate. The next higher level is the letter level containing familiar letter codes, and here familiar letters are processed faster than unfamiliar letters. Processing at the letter level is indicated in the letter condition of Figure 6 and in the letter list condition of
Figure 3, where cluster and letter pairs are processed similarly. The next level of the hierarchy contains representations of familiar letter pairs. The data from the cluster list condition of Figure 3 represent processing at this level. However, given the data from Experiment 1 , the cluster and word codes of the hierarchy do not seem to be located at separate levels.

The problem of interrelating levels of processing is discussed in other papers (LaBerge, 1976, 1977) in which a variety of perceptual processing models, hierarchical as well as nonhierarchical, are compared. However, regardless of which type of levels model is assumed, it would seem, in view of the present set of findings, that additional structures are required to represent the selective effects of context on the processing of stimulus patterns at particular levels.

\section{REFERENCES}

Aderman, D., \& Smith, E. E. Expectancy as a determinant of functional units in perceptual recognition. Cognitive Psychology, 1971, 2, 117-129.

BARON, J., \& ThURSTON, I. An analysis of the word-superiority effect. Cognitive Psychology, 1973, 4, 204-228.

Estes, W. K. Memory, perception, and decision in letter identification. In R. L. Solso (Ed.), Information processing and cognition: The Loyola symposium. New York: Wiley, 1975.

GiBson, E. J.. \& LEVIN, H. The psychology of reading. Cambridge: M.I.T. Press, 1975.

LABERGE, D. Effect of type of catch trial upon generalization gradients of reaction time. Joumal of Experimental Psychology, $1971,87,225-228$.

LABERGE, D. Attention and the measurement of perceptual learning. Memory \& Cognition, 1973, 1, 268-276.

LaBerge, D. Perceptual learning and attention. In W. K. Estes (Ed.), Handbook of learning and cognitive processes (Vol. 4). Hillsdale, N. J: Lawrence Erlbaum, 1976, in press.

LABERGE, D. The perception of units in beginning reading. In L. B. Resnick \& P. A. Weaver (Eds.), Theory and practice of early reading. Hillsdale, N.J: Lawrence Erlbaum, 1977, in press.

Laberge, D., Petersen, R. J., \& Norden, M. J. Exploring the limits of cueing. In S. Dornic (Ed.), Attention and performance (Vol. 6). London: Academic Press, 1977.

LaBerge, D., \& Samuels, S. J. Toward a theory of automatic information processing in reading. Cognitive Psychology, 1974, 6, 293-323.

Pollatsek, A., Well, A. D., \& Schindler, R. M. Familiarity affects visual processing of words. Journal of Experimental Psychology: Human Perception and Performance, 1975, 1, 328-338.

Posner, M. I., \& Mrtchell, R. F. Chronometric analysis of classification. Psychological Review, 1967, 74, 392-409.

Reicher, G. M. Perceptual recognition as a function of meaningfulness of stimulus material. Journal of Experimental Psychology, 1969, 81, 275-280.

WheEler, D. D. Processes in word recognition. Cognitive Psychology, 1970, 1, 59-85.

(Received for publication July 26, 1976; revision accepted September 20, 1976.) 